\title{
Editorial
}

\section{Modeling and Characterization of the Interaction of Electromagnetic Wave with Nanocomposites and Nanostructured Materials}

\author{
Christian Brosseau \\ Département de Physique, Université de Bretagne Occidentale, 29238 Brest Cedex 3, France
}

Received 17 December 2007; Accepted 17 December 2007

Copyright (c) 2007 Christian Brosseau. This is an open access article distributed under the Creative Commons Attribution License, which permits unrestricted use, distribution, and reproduction in any medium, provided the original work is properly cited.

\begin{abstract}
Nanostructured materials represent a size limit of the miniaturization trend of current technology. Interest in nanophases has expanded as investigators have recognized that many of the properties of finely divided matter strongly depend on the interfacial properties of the constituents by virtue of the high fraction of the overall material which is in the vicinity of an interface as well as of the confinement of electrons, excitons, and photons in small volumes. One of the interesting and important issues in predicting and understanding nanostructures and their functional behaviors is whether the properties of matter evolve gradually from bulk, as system size is reduced, and what determines this evolution behavior.

The electromagnetic characterization of nanomaterials can be considered a major part of the emerging field of nanotechnology. The potentially profound implications both for the transport properties and optics are only beginning to be explored. In that respect, multicomponent magnetic nanophases are of significant technological interest, that is, they can be considered as prospective granular magnetic films for tunable or nonreciprocal millimeter wave devices for monolithic microwave integrated circuit applications. This has also stimulated studies of the magnetoelectric effect, that is, the polarization of a material in an applied field or an induced magnetization in an external electric field.

The practical importance and industrial interest in these materials demand optimization of several types of properties in these materials. These properties include polarization, magnetization, and stability of the materials to mechanical, electrical, and magnetic fields applied during processing and operation. One of the fundamental goals of this field should be the understanding of the relationships of these properties on the composition, particle size and boundaries variations, defect structure and separation of the residual pores, but in most cases they are not well-understood.
\end{abstract}


properties is an important engineering and scientific concern. Both the editor and contributors of this volume would feel well rewarded if this issue helps relieve some of the problems of finding useful information on the morphological and electromagnetic properties of nanostructures.

Christian Brosseau 

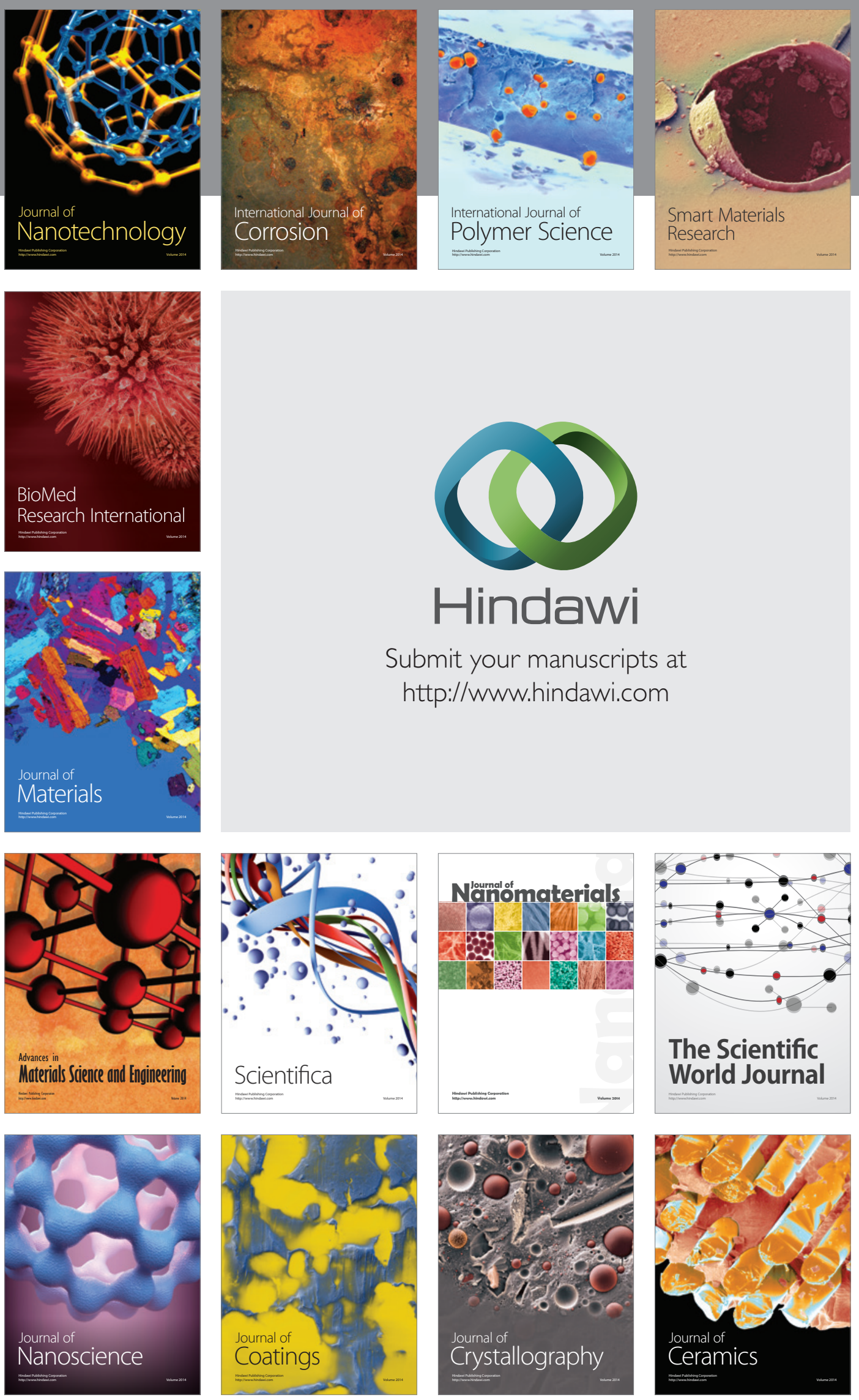

The Scientific World Journal

Submit your manuscripts at

http://www.hindawi.com

\section{World Journal}

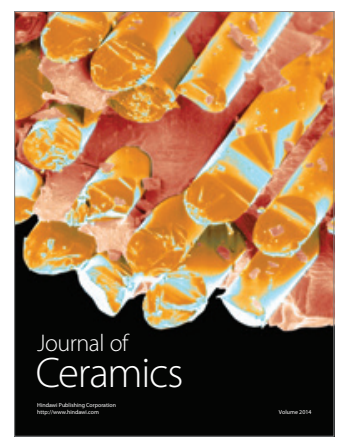

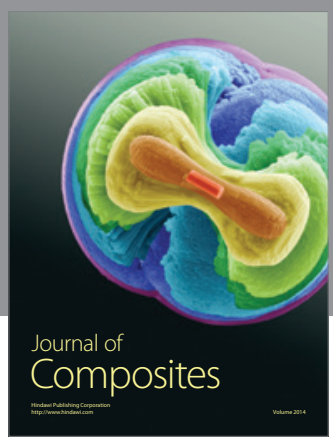
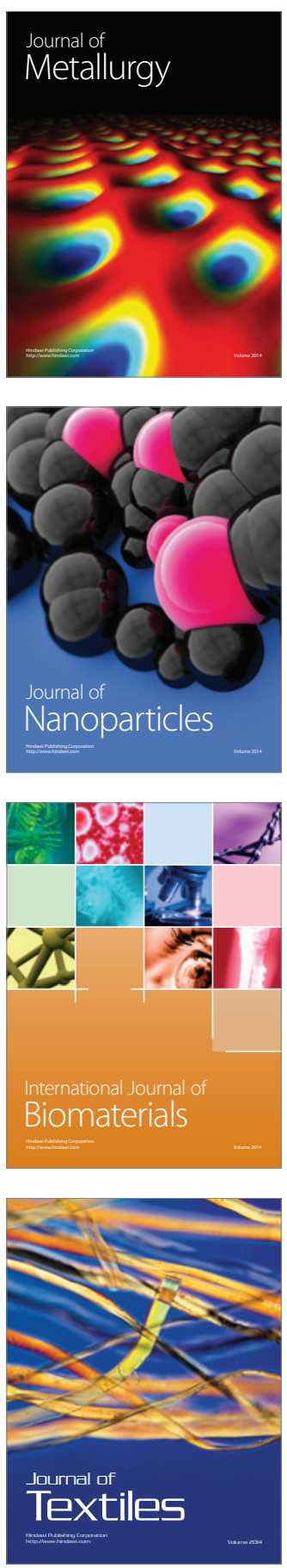